\title{
Kinetic growth walks on complex networks
}

\author{
Carlos P. Herrero ${ }^{1}$ \\ ${ }^{1}$ Instituto de Ciencia de Materiales, Consejo Superior de Investigaciones \\ Científicas (CSIC), Campus de Cantoblanco, 28049 Madrid, Spain
}

(Dated: July 6, 2018)

\begin{abstract}
Kinetically grown self-avoiding walks on various types of generalized random networks have been studied. Networks with short- and long-tailed degree distributions $P(k)$ were considered $(k$, degree or connectivity), including scale-free networks with $P(k) \sim k^{-\gamma}$. The long-range behaviour of selfavoiding walks on random networks is found to be determined by finite-size effects. The mean self-intersection length of non-reversal random walks, $\langle l\rangle$, scales as a power of the system size $N:\langle l\rangle \sim N^{\beta}$, with an exponent $\beta=0.5$ for short-tailed degree distributions and $\beta<0.5$ for scale-free networks with $\gamma<3$. The mean attrition length of kinetic growth walks, $\langle L\rangle$, scales as $\langle L\rangle \sim N^{\alpha}$, with an exponent $\alpha$ which depends on the lowest degree in the network. Results of approximate probabilistic calculations are supported by those derived from simulations of various kinds of networks. The efficiency of kinetic growth walks to explore networks is largely reduced by inhomogeneity in the degree distribution, as happens for scale-free networks.
\end{abstract}

PACS numbers: 89.75.Fb, 87.23.Ge, 05.40.Fb, 89.75.Da

\section{INTRODUCTION}

The past few years have seen extraordinary progress in the description of real-life complex systems in terms of networks or graphs, where nodes represent typical system units and edges represent interactions between connected pairs of units. Such a topological description has been applied for modelling several kinds of natural and man-made systems, and is currently employed to study different processes taking place on real systems (social, biological, technological, economic) [1, 2, 3, 4]. Two highlights of these developments are the Watts-Strogatz small-world networks [5] and the so-called scale-free networks [6], which incorporate various aspects of real systems. In particular, they are characterized by the fact that the average separation between sites increases with system size $N$ not faster than $\log N$. These complex networks provide us with the underlying topological structure to analyze processes such as spread of infections [7, 8], signal propagation [5, 9, 10], and random spreading of information [11, 12].

In the last years, researchers have been accumulating evidence [13, 14, 15, 16] that several kinds of networks possess a degree (or connectivity, $k$ ) distribution given by a power law, $P_{\mathrm{SF}}(k) \sim k^{-\gamma}$, with an exponent $\gamma$ usually in the range $2<\gamma<3$ [2, 17]. The origin of such power-law degree distributions was addressed by Barabási and Albert [6], who argued that two ingredients are sufficient to explain the scale-free character of many real-life networks, namely: growth and preferential attachment. They found that a combination of both criteria yields non-equilibrium scale-free networks with an exponent $\gamma=3$.

Social networks form the substrate where dynamical processes such as information spreading and disease propagation take place 2]. These networks have the property of being able to find a target quickly (they are "searchable") [18, 19, 20], as a consequence of their topological characteristics. To understand several dynamical processes (diffusion, navigation, search) on complex networks, several authors have analyzed various properties of random walks on these networks 12, 21, 22, 23, 24, 25, 26, 27]. These studies give us valuable information on dynamical processes in real systems, in spite of the fact that actual processes are usually neither purely random nor totally deterministic.

In contrast with unrestricted random walks, selfavoiding walks (SAWs) on a given network cannot return to sites visited earlier in the same walk, and one can expect the latter to be more effective for search and exploration. In fact, this kind of walks have been used to propose local search strategies in scale-free networks 28. However, the self-avoiding condition causes attrition, in the sense that a large fraction of paths generated at random have to be abandoned because they overlap 29, 30]. This fact can limit appreciably the capability of SAWs for exploring real-life networks.

SAWs on regular lattices have been employed since many years for modelling structural and dynamical properties of macromolecules 31, 32], as well as to characterize complex crystal structures 33 and to study critical phenomena 34]. Several universal constants for SAWs on lattices are now well known 35]. In our context, SAWs were studied earlier in small-world networks [36], and have been also employed to obtain the so-called $L$ expansions of complex networks [37]. Recently, kinetic growth walks on uncorrelated scale-free networks were considered, with special emphasis upon the influence of attrition on the maximum length of the paths [30]. It was found that the average length scales as a power of the system size, with an exponent that depends on the characteristics of the considered networks.

Scale-free networks have a notoriously inhomogeneous distribution of degrees, and it is not yet clear whether properties of SAWs on these networks are due to that large inhomogeneity, or are general of (uncorrelated) 
complex networks with arbitrary degree distributions [38]. Here we study kinetically grown walks on random networks with constant degree (regular graphs) and with short-tailed degree distributions, and discuss the "attrition problem" on these networks. We obtain the number of surviving walks to a given length $n$ by an approximate analytical procedure, and the results are compared with those derived from simulations of different kinds of networks. Results for networks with short-tailed degree distributions are in turn compared with those found for scale-free networks. We note that the term length is employed throughout this paper to indicate the (dimensionless) number of steps of a walk, as is usual in the literature on networks [2].

The paper is organized as follows. In Sec. II we give some general concepts related to SAWs, along with definitions of the kinetic growth walks considered here. Sec. III is devoted to study the self intersection and attrition of growing walks in networks with constant connectivity. The same properties are studied for walks on networks with short-tailed and scale-free degree distributions in Secs. IV and V respectively. The paper closes with a Discussion in Sec. VI.

\section{DEFINITIONS AND METHOD}

A self-avoiding walk is defined as a walk along the links of a network which cannot intersect itself. In each step the walk is restricted to moving to a nearest-neighbour node, and the self-avoiding condition constrains the walk to occupy only sites which have not been visited earlier in the same walk. To study several kinds of dynamic processes, such as navigation on networks, one can consider kinetically grown walks, for which a temporal sequence is assumed.

Here we will consider two kinds of growing walks. The first kind will be non-reversal walks [29]. In these walks one randomly chooses the next step from among the neighbouring nodes, excluding the previous one. If one selects a node visited previously, then the walk stops (see Fig. 1). These walks will be used to study the selfintersection length. The second kind of walks considered here are kinetic growth walks (KGWs) [39], in which one randomly chooses the next step among the neighbouring unvisited sites and stops growing when none are available. These walks were employed to describe the irreversible growth of linear polymers [39, 40], and will be used here to study the attrition length of walks on various kinds of networks. KGWs are less sensitive to attrition than non-reversal walks, in the sense that in the former the walker always escapes whenever a way exists (see Fig. 1 ). Note that both kinds of walks are kinetically grown, but we use the expression 'kinetic growth walks' to denote those of the second type, as usually done in the literature [39, 41]. We call 'non-reversal walks' those of the first type, to emphasize the fact that the non-reversal condition is the only restriction on this kind of walks,

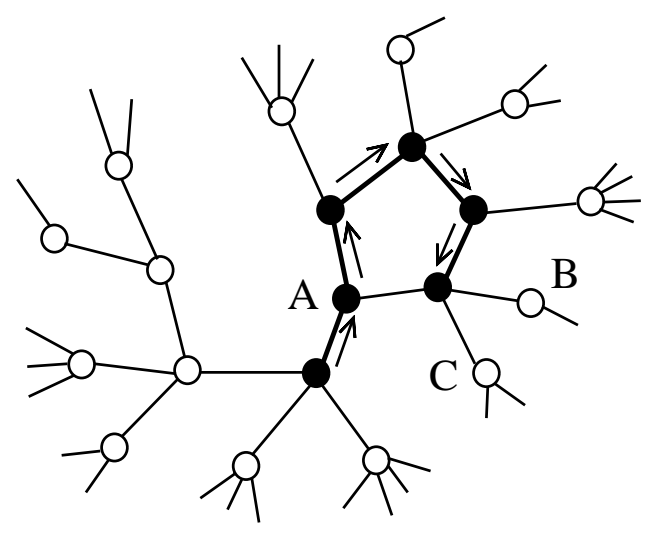

FIG. 1: Schematic diagram showing a non-reversal walk of length $n=5$ on a realization of a random graph. Open and black circles represent unvisited and visited nodes respectively. The non-reversal condition allows in principle for the next (sixth) step three possible nodes (denoted $A, B$, and $C)$. For a non-reversal walk one chooses among nodes $A, B$, and $C$. If $A$ is selected, then the walk stops. For a kinetic growth walk, one chooses $B$ or $C$, each with $50 \%$ probability.

until they reach a node visited earlier. Both ensembles consist of the same set of walks as SAWs, but each has a weight depending on its growth process. In particular, for networks in which all nodes have the same degree (regular networks), our non-reversal walks coincide with usual self-avoiding walks, in the sense that all walks of a given length have the same weight.

To analyze the effect of inhomogeneity in the degree distribution on the characteristics of self-avoiding walks, we will consider three kinds of networks: (1) random networks with constant degree, (2) networks with shorttailed degree distribution, and (3) scale-free networks, with a power-law distribution of degrees. For simulations we have generated networks with various sizes $N$ and mean connectivities $\langle k\rangle$. To generate a network, we first define the number of nodes $N_{k}$ with degree $k$, following a probability distribution $P(k)$; second, we ascribe a degree to each node according to the set $\left\{N_{k}\right\}$, and finally we connect randomly ends of links (giving a total of $L=$ $\sum_{k} k N_{k} / 2$ connections), with two conditions: (i) no two nodes can have more than one bond connecting them, and (ii) no node can be connected by a link to itself. All networks studied here contain a single component, i.e. any node in a network can be reached from any other node in a finite number of steps. For a given kind of networks, once fixed the parameters defining the degree distribution, we considered several network realizations, and for a given network we took randomly the starting nodes for the walks. In each case considered, the total number of walks amounted to $5 \times 10^{5}$.

In general, for a given length $n$, the number of different SAWs on a network changes with the starting node. We will call $s_{n}$ the average number of SAWs of length $n$, i.e. the mean value obtained by averaging over the network sites and over different network realizations. For Erdös- 
Rényi (ER) random graphs with Poissonian distribution of degrees, one has $s_{n}^{r d}=\langle k\rangle^{n}$ 36]. For walks of length $n \ll N$ in generalized random networks, one has 30]

$$
s_{n}=\langle k\rangle\left(\frac{\left\langle k^{2}\right\rangle}{\langle k\rangle}-1\right)^{n-1} .
$$

It is known that the number of SAWs on regular lattices scales for large $n$ as $s_{n} \sim n^{\Gamma-1} \mu^{n}$, where $\Gamma$ is a critical exponent which depends on the lattice dimension $D$ and $\mu$ is the so-called connective constant. For $D>4$ one has $\Gamma=1$ [29, 35]. The connective constant can be obtained from the large- $n$ limit of the ratio $s_{n} / s_{n-1}$, which in general depends on $n$. This ratio becomes independent of $n$ for random networks when the system size $N \rightarrow \infty$. This happens because for large $N$ the probability of finding loops with $n^{\prime} \leq n$ in a $n$-step walk is negligible, and the self-avoiding condition does not impose in practice any restriction on non-reversal walks. Thus, for large $N$ the connective constant $\mu_{\infty}$ for random networks is $\mu_{\infty}=\left\langle k^{2}\right\rangle /\langle k\rangle-1$. (Note that $\mu_{\infty}$ diverges for diverging $\left\langle k^{2}\right\rangle$, as happens for scale-free networks with $\gamma \leq 3$.) For finite networks, however, there appear loops of any size [42], and $s_{n}$ will be lower than given by Eq. (11). These finite-size corrections will be of order $n / N$ for $n / N \ll 1$. The effects of this reduction in the number of non-reversal and kinetic growth walks on random networks will be considered in the following Sections.

\section{REGULAR RANDOM NETWORKS}

Here we consider random networks with constant connectivity $k>2$. These are the so-called regular graphs, in which all nodes have the same degree [43]. Regular graphs with $k=2$ are made up by a set of disjointed rings, and will not be considered here. Regular random networks have been employed for modelling disordered systems, such as spin glasses [44, 45]. We consider first this kind of networks, since for them the probabilistic calculations presented below are somewhat simpler than for networks with dispersion in the degree distribution.

\section{A. Self-intersection length}

To study the probability of a walk intersecting itself, we consider non-reversal walks that stop when they reach a node already visited in the same walk. The number of steps of a given walk before intersecting itself will be called self-intersection length and will be denoted $l$.

In order to obtain the mean self-intersection length of non-reversal walks, we will calculate first the conditional probability $p_{n}$ of a visited node being found in step $n+1$, assuming that the walk has in fact reached step $n(1 \ll$ $n \ll N)$. After $n$ steps, the number of visited nodes is $n$, and that of unvisited ones is $N-n$. Thus, the number of ends of links connected to visited and unvisited nodes is

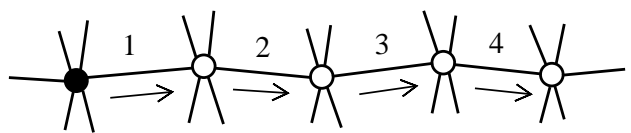

FIG. 2: Schematic representation of a kinetically grown walk on a network with constant connectivity $(k=6)$. A black circle indicates the starting node and labels show the step number. For each visited node (apart from the first and the last one), there remain $k-2$ links which have not yet been used in the walk.

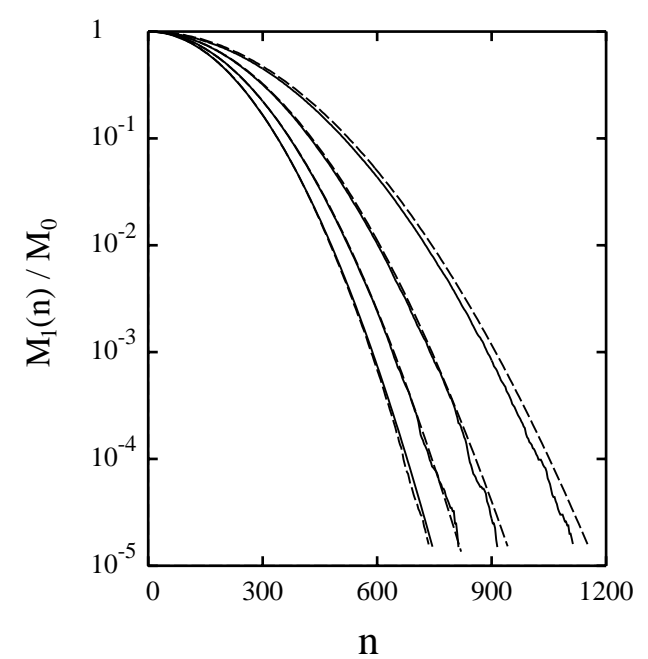

FIG. 3: Fraction of non-reversal walks that survive after $n$ steps, without intersecting themselves. Results are plotted for regular random networks with different degrees and size $N=20000$ nodes. From left to right: $k=10,6,4$, and 3 . Solid and dashed lines indicate results of network simulations and analytical calculations, respectively.

$v=(k-2) n$ and $u=k(N-n)$, respectively. This is due to the fact that a visited node has $k-2$ possible links to reach it, as two of its connections are not available because they were employed earlier: one for an incoming step and one for an outgoing step (see Fig. 2). Hence,

$$
p_{n}=\frac{v}{v+u}=\frac{(k-2) n}{k N-2 n} .
$$

Then, one has

$$
p_{n}=w \frac{n}{N}+O\left(\left[\frac{n}{N}\right]^{2}\right),
$$

with $w=(k-2) / k$. In the following, only terms linear in $n / N$ will be retained.

Let us now consider $M_{0}$ non-reversal walks starting from nodes taken at random, and call $M_{1}(n)$ the number of walks surviving after $n$ steps (i.e., those which did not arrive at any node visited earlier). Then,

$$
M_{1}(n)-M_{1}(n+1)=p_{n} M_{1}(n),
$$


which can be solved by iteration with the initial condition $M_{1}(0)=M_{0}$. An analytical expression for $M_{1}(n)$ can be found by dealing with $n$ as a continuous variable $x$, and writting a differential equation for $M_{1}(x)$ :

$$
\frac{d M_{1}}{d x}=-\frac{w}{N} x M_{1}
$$

so that, for integer $n$ :

$$
M_{1}(n)=M_{0} \exp \left(-\frac{w}{2 N} n^{2}\right) .
$$

In Fig. 3 we show the fraction of remaining walks, $M_{1}(n) / M_{0}$, as a function of $n$ for networks with different connectivities. Dashed lines were obtained by using Eq. (6) and solid lines correspond to results of simulations. The agreement between both sets of results is good, and we observe that it is better the larger the connectivity ( $k$ increases from right to left).

The probability distribution $R(l)$ for the selfintersection length $l$, given by $R(l) \equiv\left[M_{1}(l)-M_{1}(l+\right.$ 1)] $/ M_{0}$, is therefore

$$
R(l)=p_{n} \frac{M_{1}(l)}{M_{0}}=\frac{w l}{N} \exp \left(-\frac{w}{2} \frac{l^{2}}{N}\right),
$$

which gives the probability of a walk returning to a visited site in step $l$. With this probability distribution we obtain the average self-intersection length:

$$
\langle l\rangle^{2} \approx \frac{\pi k N}{2(k-2)} .
$$

For the dispersion in the self-intersection length of the walks, one has from Eq. (7) $\sigma_{l}^{2}=C k N /(k-2)$, with a constant $C=2-\pi / 2 \approx 0.43$.

\section{B. Attrition length}

We now consider KGWs, that stop when they arrive at a node (called hereafter blocking node) in which they cannot continue because all neighbouring nodes have been already visited. The number of steps of a given walk until being blocked will be called attrition length of the walk, and will be denoted $L$.

We will calculate the mean attrition length of KGWs, and obtain its asymptotic dependence for large system size $N$. With this purpose, we will derive a probability distribution for $L$, in a manner similar to that used above for the self-intersection length. The probability of a KGW reaching a blocking node in step $n$ is that of finding a node for which all its links except one (employed for an incoming step) connect it with nodes previously visited. Then, the average number $N^{\prime}$ of blocking nodes is given by the binomial distribution

$$
N^{\prime}=k N p_{n}^{k-1}\left(1-p_{n}\right) \approx k N p_{n}^{k-1},
$$

where $p_{n}(\ll 1)$ is the average fraction of links joining a node with nodes visited in a walk, as given in Eq. (3).
Since there is one link leading to each possible blocking node, the probability $q_{n}$ of finding one of these nodes in step $n+1$ is $q_{n}=N^{\prime} / N_{\text {end }}$, where $N_{\text {end }}$ is the average number of possible ends of links at step $n+1$. This number is $N_{\text {end }}=k N-k n$, because for each visited node one has $k$ unavailable ends of links. Hence, for $n \ll N$ we have $N_{\text {end }} \approx k N$, and $q_{n} \approx p_{n}^{k-1}$.

We now consider $M_{0}$ kinetic growth walks and use the probability $q_{n}$ to calculate the number $M_{2}(n)$ of surviving walks to length $n$. $M_{2}(n)$ can be obtained from the difference

$$
M_{2}(n)-M_{2}(n+1)=q_{n} M_{2}(n),
$$

which gives the number of walks finishing at step $n$. Dealing with $n$ as a continuous variable $x$, we have a differential equation:

$$
\frac{d M_{2}}{d x}=-\left(\frac{w}{N}\right)^{k-1} x^{k-1} M_{2}
$$

with $w=(k-2) / k$ (see above). Then, for integer $n$ we have

$$
M_{2}(n)=M_{0} \exp \left[-\left(\frac{n}{x_{0}}\right)^{k}\right],
$$

and $M_{2}(n) / M_{0}$ gives the probability of surviving to length $n$. Here $x_{0}$ is a constant characteristic of the considered networks, given by $x_{0}=k[N /(k-2)]^{1-1 / k}$.

Consequently, the probability distribution $Z(L)$ for the attrition length of these walks, given by $Z(L) \equiv\left[M_{2}(L)-\right.$ $\left.M_{2}(L+1)\right] / M_{0}$, is

$$
Z(L)=q_{L} \frac{M_{2}(L)}{M_{0}}=p_{L}^{k-1} \exp \left[-\left(\frac{L}{x_{0}}\right)^{k}\right] .
$$

From this distribution, we obtain a mean attrition length

$$
\langle L\rangle \approx x_{0} \Gamma\left(\frac{k+1}{k}\right),
$$

where $\Gamma$ is Euler's gamma function. Thus, the dependence of $\langle L\rangle$ on $N$ for large systems is controlled by $x_{0}$. Indeed, $x_{0} \sim N^{\alpha}$, with an exponent $\alpha=1-1 / k$ ranging from $\alpha=2 / 3$ for $k=3$ to $\alpha=1$ for large $k(k \rightarrow \infty)$.

The mean attrition length is plotted in Fig. 4 as a function of system size $N$ for several connectivities $k$. The lines were obtained by using Eq. (14), and symbols are data points derived from simulations ( $k$ increases from right to left). The approximate expression (14) predicts values of $\langle L\rangle$ close to the actual ones for this kind of networks. Note that for a given size $N,\langle L\rangle$ increases as $k$ rises. However, for the mean self-intersection length presented above, $\langle l\rangle$ decreases for rising $k$.

\section{RANDOM NETWORKS WITH SHORT-TAILED DEGREE DISTRIBUTION}

Classical random networks are the well-known ER random graphs, with Poissonian distribution of degrees [43]. 


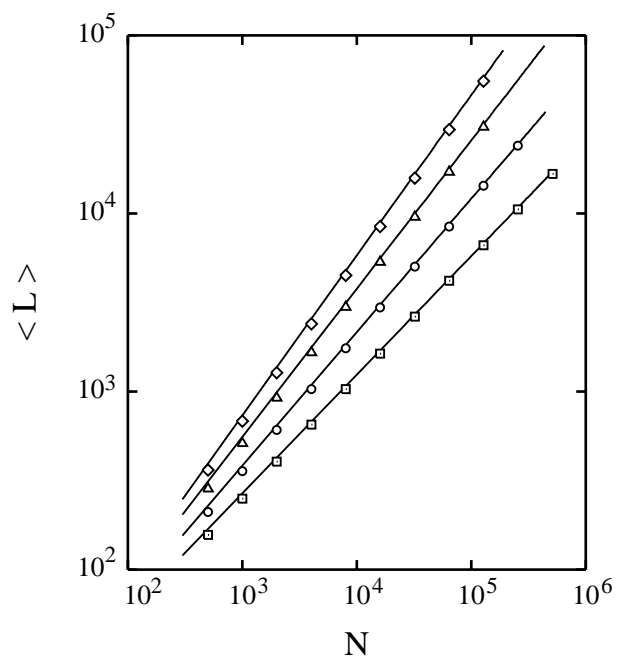

FIG. 4: Mean attrition length $\langle L\rangle$ as a function of system size $N$ for regular random networks with different degrees. Symbols represent simulation results: squares, $k=3$; circles, $k=4$; triangles, $k=6$; diamonds, $k=10$. Error bars are less than the symbol size. Lines indicate results of analytical calculations.

This means that the degree distribution is short-tailed, since it decreases for large $k$ as $1 / k !$. These networks contain nodes with $k=0$ (isolated nodes) and with $k=1$. Isolated nodes are never reached in a walk, unless they are the starting node, in which case the walk cannot proceed. This is not a major problem, as it is equivalent to a renormalization of the number of walks. However, nodes with $k=1$ behave as culs-de-sac for KGWs. In fact, a KGW arriving at a node with connectivity $k=1$ cannot continue, even though it has not yet intersected itself. For this reason, we will consider networks similar to ER graphs, but with the minimum degree $k_{0}>1$. In particular, they will have the following distribution of degrees:

$$
P_{\mathrm{sh}}(k)=\frac{\lambda^{k-k_{0}}}{\left(k-k_{0}\right) !} e^{-\lambda}
$$

for $k \geq k_{0}$, and $P_{\mathrm{sh}}(k)=0$ for $k<k_{0}$. Such a connectivity distribution can be realized by distributing first $N k_{0} / 2$ links in such a way that each node has $k_{0}$ connections (as for networks in the previous Section), and then linking pairs of nodes with a certain probability $a$. This probability is related with the parameter $\lambda$ by $a=\lambda /(N-1)$, as in ER graphs. For $k_{0}=0$, we recover ER graphs with a Poissonian distribution of degrees.

\section{A. Self-intersection length}

To calculate the mean self-intersection length of nonreversal walks, we will proceed in a way similar to the case of regular random networks, but taking now into account the presence of nodes with different degrees. We consider first nodes with a given connectivity $k$. The probability $Q(k)$ of arriving at a node with this degree is proportional to $k$, i.e.: $Q(k)=k P(k) /\langle k\rangle$, where $\langle k\rangle$ is a normalization factor. Then, the average number of nodes with degree $k$ visited in an $n$-step non-reversal walk is

$$
V_{k}=n Q(k),
$$

and the mean number of nodes yet unvisited is $U_{k}=$ $N_{k}-V_{k}$, or

$$
U_{k}=N P(k)-n Q(k) .
$$

Thus, the number of ends of links connected to visited and unvisited nodes with degree $k$ is $(k-2) V_{k}$ and $k U_{k}$, respectively (see Fig. 2). Therefore, the conditional probability $p_{n}$ of finding a visited node with any degree in step $n+1$ (assuming that the walk actually reached step $n$ ) is

$$
p_{n}=\frac{\sum_{k}(k-2) V_{k}}{\sum_{k}\left[(k-2) V_{k}+k U_{k}\right]} .
$$

Inserting here expressions (16) and (17) for $V_{k}$ and $U_{k}$, we obtain

$$
p_{n}=\frac{n}{\langle k\rangle} \frac{\left\langle k^{2}\right\rangle-2\langle k\rangle}{\langle k\rangle N-2 n} .
$$

For $n \ll N$, one can approximate to order $n / N$ :

$$
p_{n} \approx w \frac{n}{N},
$$

with

$$
w=\frac{\left\langle k^{2}\right\rangle-2\langle k\rangle}{\langle k\rangle^{2}} .
$$

This expression is general for random networks. A particular case is that of regular networks with connectivity $k$, for which $w=(k-2) / k$ (see above). For the distribution $P_{\mathrm{sh}}(k)$, we have $\langle k\rangle=\lambda+k_{0}$ and $\left\langle k^{2}\right\rangle=\lambda+\left(\lambda+k_{0}\right)^{2}$. Then, to have $w>0$ it is sufficient $\lambda>1$ or $\langle k\rangle>2$. We note that the inequality $\left\langle k^{2}\right\rangle-2\langle k\rangle>0$, which gives $w>0$, is a necessary condition to have a giant component in a network [46].

To calculate the probability distribution for the selfintersection length $l$, we proceed as in the previous Section, from Eq. (4) to Eq. (7). In particular, for the number of surviving walks $M_{1}(n)$ we find the same expression (6) with $w$ given in Eq. (21). The fraction of surviving walks $M_{1}(n) / M_{0}$ is shown in Fig. 5 for networks with $\lambda=4, k_{0}=2$, and several system sizes, with $N$ increasing from left to right. Dashed and solid lines indicate results of analytical calculations and network simulations, respectively. For the mean self-intersection length we find

$$
\langle l\rangle^{2} \approx \frac{\pi N}{2 w},
$$




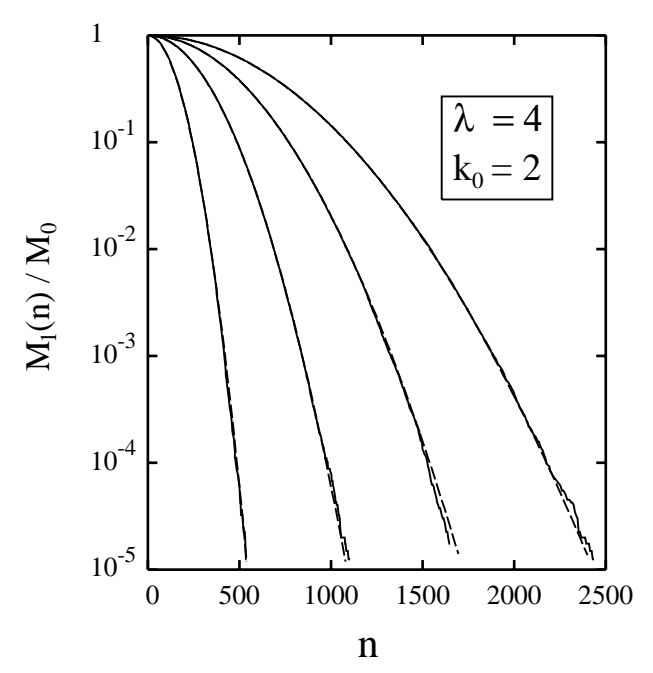

FIG. 5: Fraction of non-reversal walks that survive after $n$ steps, without intersecting themselves. Results are plotted for random networks with lowest degree $k_{0}=2$ and $\lambda=4$, and several system sizes. From left to right: $N=10^{4}, 4 \times 10^{4}, 10^{5}$, and $2 \times 10^{5}$. Solid and dashed lines correspond to results of network simulations and analytical calculations, respectively.

which is a generalization of Eq. (8) to random networks with arbitrary distribution of degrees. This mean value scales with system size as $\langle l\rangle \sim N^{1 / 2}$, regardless of the details of the degree distribution (here, parameters $k_{0}$ and $\lambda$ ). These details affect the parameter $w$ appearing in Eq. (22), but not the functional form.

\section{B. Attrition length}

We will now calculate the mean attrition length of KGWs, and obtain its asymptotic dependence for large system size $N$, similarly to the case of regular random networks treated in section III B. For a given degree $k$, the average number $U_{k}$ of unvisited nodes in an $n$-step walk is given by Eq. (17). Among these nodes, the number $N_{k}^{\prime}$ of possible blocking nodes (those with $k-1$ unavailable links and one available connection) is given by the binomial distribution:

$$
N_{k}^{\prime}=k U_{k} p_{n}^{k-1}\left(1-p_{n}\right),
$$

where $p_{n}$ is the average fraction of links joining a node with visited nodes, as given in Eq. (20).

For each possible blocking node there is one (incoming) link available for the walk. Then, the probability of finding one of these nodes in step $n+1$ is

$$
q_{n}=\frac{1}{N_{\text {end }}} \sum_{k \geq k_{0}} N_{k}^{\prime},
$$

$N_{\text {end }}$ being the average number of possible ends of links for step $n$, given by $N_{\text {end }}=\langle k\rangle N-n\langle k\rangle_{Q}$ [the subscript $Q$ indicates average with the distribution $Q(k)$, as nodes with degree $k$ are visited with probability $Q(k) \propto k P(k)]$.
For large enough $N$ (small enough $p_{n}$ ), whenever $N_{k} p_{n}^{k-k_{0}} \ll N_{k_{0}}$, one has $N_{k}^{\prime} \ll N_{k_{0}}^{\prime}$. If this inequality is true for all $k>k_{0}$, then the probability $q_{n}$ can be approximated to order $n / N$ as:

$$
q_{n} \approx \frac{N_{k_{0}}^{\prime}}{\langle k\rangle N} \quad(n \ll N),
$$

with $N_{k_{0}}^{\prime} \approx k_{0} N_{k_{0}} p_{n}^{k_{0}-1}$. In such a case, the calculation of the number $M_{2}(n)$ of walks surviving to length $n$ is greatly simplified, since one can write the difference given in Eq. (10) as a differential equation:

$$
\frac{d M_{2}}{d x}=-T x^{k_{0}-1} M_{2},
$$

with the network-dependent parameter $T=$ $N_{k_{0}} k_{0} w^{k_{0}-1} /\left(N^{k_{0}}\langle k\rangle\right)$ and $w$ given in Eq. (21). Following as above in section III.B, we find for the probability distribution $Z(L)$ of the attrition length:

$$
Z(L)=T L^{k_{0}-1} \exp \left[-\left(\frac{L}{x_{0}}\right)^{k_{0}}\right],
$$

where $x_{0}$ is a constant characteristic of the considered network, given by $x_{0}^{k_{0}}=k_{0} / T$. The shape of this distribution coincides with that found earlier for uncorrelated scale-free networks 30. In fact, it is general for random networks verifying Eq. (25), and reduces to Eq. (13) in the case of networks with constant degree, by inserting the appropriate expressions for $x_{0}$ and $T$. The distribution $Z(L)$ is strongly dependent on the lowest degree $k_{0}$, because nodes with this degree control in fact the maximum length of KGWs in these networks. Note that contrary to non-reversal walks, the length of KGWs studied here can be in some cases on the order of the network size $N$, and then the condition $n \ll N$ leading to Eqs. (25) and (26) may be not fulfilled. In such a case, one has to employ the general expression for $q_{n}$ given in Eq. (24) and iterate Eq. (10).

The distribution $Z(L)$ gives a mean attrition length

$$
\langle L\rangle \approx \frac{x_{0}}{k_{0}} \Gamma\left(\frac{1}{k_{0}}\right),
$$

$\Gamma$ being Euler's gamma function. Thus, for a given lowest degree $k_{0}$, the dependence of $\langle L\rangle$ on $N$ for large systems is controlled by $x_{0}$. To obtain the asymptotic dependence of $x_{0}$, we note that the parameter $T$ behaves as $N^{1-k_{0}}$ for $N \rightarrow \infty$, and therefore $x_{0}$ and $\langle L\rangle$ increase for large $N$ as $N^{1-1 / k_{0}}$. Expression (28) is similar to that found for the mean attrition length in regular random networks, Eq. (14). In fact, the latter is a particular case of the former, i.e., it corresponds to a short-tailed degree distribution with $\lambda=0$ (in this case $P_{\mathrm{sh}}(k)=\delta_{k, k_{0}}$ ).

Shown in Fig. 6 is the mean attrition length $\langle L\rangle$ vs system size $N$ for networks with $k_{0}=3$ and three $\lambda$ values. Results derived from network simulations (symbols) follow closely those yielded by using the probability $q_{n}$ 


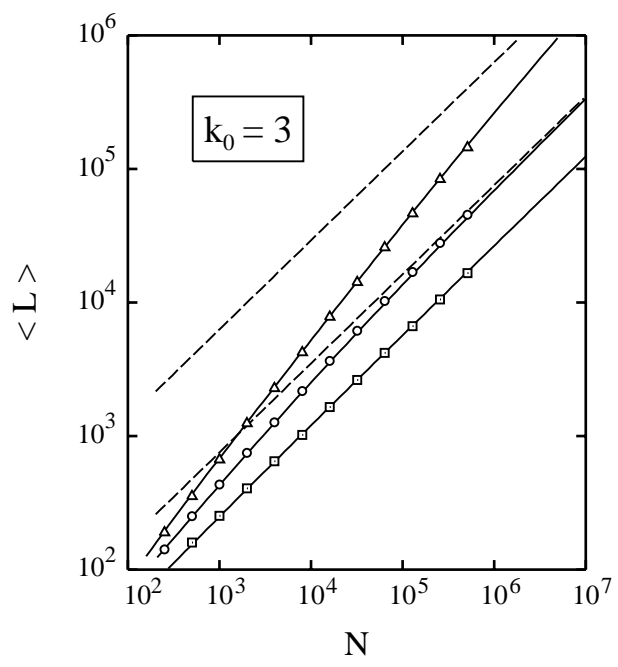

FIG. 6: Mean attrition length $\langle L\rangle$ as a function of system size for random networks with minimum degree $k_{0}=3$ and different values of $\lambda$. Symbols indicate simulation results: squares, $\lambda=0$; circles, $\lambda=4$; triangles, $\lambda=10$. Error bars are less than the symbol size. Solid lines were obtained by iteration of Eq. (10) with the probability $q_{n}$ given in Eq. (24). Dashed lines correspond to the asymptotic large- $N$ limit given by Eq. (28).

in Eq. (24) to iterate Eq. (10) (solid lines). Dashed lines correspond to Eq. (28), which is the asymptotic limit for $\langle L\rangle$ at large $N$. For $\lambda=0$ (regular random networks with $k=3$ ), it is indistinguishable from the solid line. For $\lambda>0$ one observes that the larger $\lambda$, the larger $N$ required for convergence between solid and dashed lines. This occurs because values of $N$ required for Eqs. (25) and (28) to be valid increase with $\lambda$. In any case, Eq. (28) describes correctly the large- $N$ limit of the mean attrition length, which for $k_{0}=3$ displays the dependence $\langle L\rangle \sim N^{1-1 / k_{0}}=N^{2 / 3}$.

\section{SCALE-FREE NETWORKS}

We now consider equilibrium scale-free networks with degree distribution $P_{\mathrm{SF}}(k) \sim k^{-\gamma}$. They are characterized, apart from the exponent $\gamma$ and the system size $N$, by the minimum degree $k_{0}$. Kinetically-grown walks on this kind of networks have been studied earlier [30]. Here we will only give the main results for the sake of completeness and comparison with those presented above for networks with constant degree and short-tailed degree distribution. One expects that the large inhomogeneity of connectivities present in scale-free networks can affect significantly the long-range behaviour of KGWs.

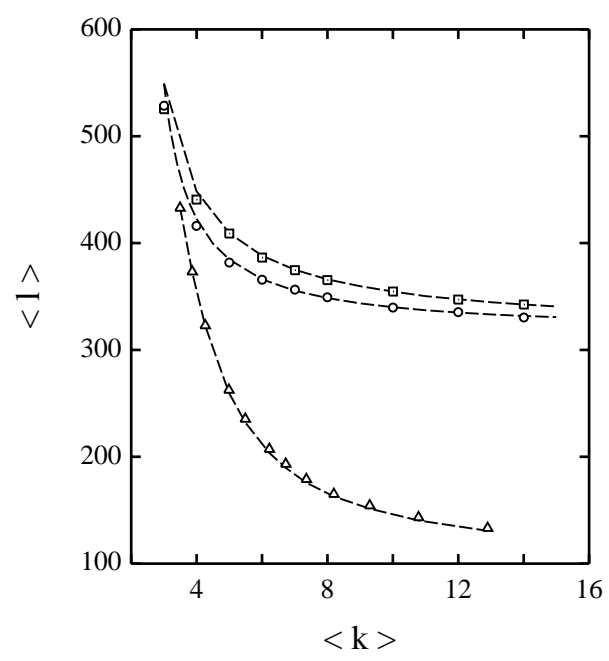

FIG. 7: Mean self-intersection length $\langle l\rangle$ vs mean connectivity for different kinds of networks with size $N=64000$ nodes. Symbols represent results of simulations: Squares, regular random networks; circles, random networks with short-tailed degree distribution $\left(k_{0}=3\right)$; triangles, scale-free networks with $k_{0}=3$ and several $\gamma$ values (between 2 and 5, from right to left). Dashed lines were obtained from Eq. (22) with the parameter $w$ corresponding to each network.

\section{A. Self-intersection length}

For uncorrelated scale-free networks, the selfintersection length of non-reversal walks can be calculated by using the expressions given in Section IV.A, which are general for random networks with arbitrary degree distribution. In particular, the mean $\langle l\rangle$ is given by Eq. (22), with $w=\left(\left\langle k^{2}\right\rangle-2\langle k\rangle\right) /\langle k\rangle^{2}$. For scalefree networks, $\langle l\rangle$ depends on the system size and on the exponent $\gamma$ of the degree distribution through the mean values $\langle k\rangle$ and $\left\langle k^{2}\right\rangle$, but does not change significantly with $k_{0}[30]$. Depending on the value of $\gamma$, one finds different trends for $w$ and $\langle l\rangle$ as functions of the system size. For large $N$ and $\gamma>2, w \sim\left\langle k^{2}\right\rangle$ and the mean self-intersection length scales as $\langle l\rangle \sim\left(N /\left\langle k^{2}\right\rangle\right)^{1 / 2}$. For $\gamma>3,\left\langle k^{2}\right\rangle$ converges to a constant, and then $\langle l\rangle \sim \sqrt{N}$, as for networks with short-tailed degree distributions.

In Fig. 7 we compare the mean self-intersection length for different kinds of networks, all with the same size $N=64000$ nodes. In this figure, we have plotted $\langle l\rangle$ as a function of the mean connectivity $\langle k\rangle$ for regular random networks (squares), networks with short-tailed degree distribution $P_{\mathrm{sh}}(k)$ (circles), and scale-free networks with several values of the exponent $\gamma$ (triangles). For $P_{\mathrm{sh}}(k)$ and $P_{\mathrm{SF}}(k)$, the lowest degree was assumed to be $k_{0}=3$. Dashed lines correspond to calculations carried out by using Eq. (22) [or its particular case, Eq. (8), for regular networks]. Note that for the distribution $P_{\mathrm{sh}}(k)$, the change in $\langle k\rangle$ is obtained by varying the parameter $\lambda$ in Eq. (15), whereas for $P_{\mathrm{SF}}(k)$ one has to change $\gamma$ when keeping constant $N$ and $k_{0}$. 


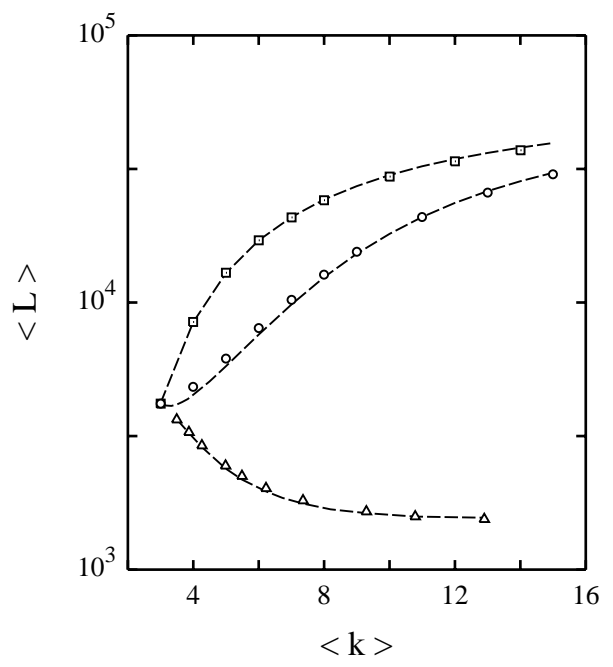

FIG. 8: Mean attrition length $\langle L\rangle$ as a function of mean connectivity for different kinds of networks with size $N=64000$ nodes. Symbols represent results of simulations: Squares, regular random networks; circles, random networks with shorttailed connectivity distribution $\left(k_{0}=3\right)$; triangles, scale-free networks with $k_{0}=3$ and $\gamma$ ranging from 2 to 5 . Dashed lines were obtained by iteration from Eq. (10) with the probability $q_{n}$ given in Eq. (24).

As commented above, $\langle l\rangle$ decreases as $\langle k\rangle$ rises. For a given $\langle k\rangle$, results for short-tailed degree distributions are slightly lower than those for regular random networks. This decrease is more appreciable for scale-free networks, especially for large $\langle k\rangle$. For constant size $N$, this is basically due to an increase in $\left\langle k^{2}\right\rangle$ as $\langle k\rangle$ is raised. In practice, the decrease in $\langle l\rangle$ is associated to the presence of nodes with large $k$, which are visited more frequently. Once visited, they are more effective to limit the length of a walk than nodes with low $k$.

\section{B. Attrition length}

For the attrition length of kinetic growth walks in uncorrelated scale free networks one can use the formulas presented in Sect. IV.B. In particular, Eq. (25) can be applied as soon as $n \ll N$, because in these networks $N_{k_{0}}>N_{k}$ for $k>k_{0}$. Then, the mean length $\langle L\rangle$ can be approximated by Eq. (28) with the networkdependent parameter $x_{0}$, which controls the behaviour of $\langle L\rangle$ for large $N$. To obtain the asymptotic dependence of $x_{0}$, we note that $N_{k_{0}} / N$ converges to a constant for large $N$. For $\gamma>2, w \sim\left\langle k^{2}\right\rangle$, and therefore $x_{0}^{k_{0}} \sim\left(N /\left\langle k^{2}\right\rangle\right)^{k_{0}-1}$. Thus, for $\gamma>3$, with $\left\langle k^{2}\right\rangle$ converging to a finite value as $N \rightarrow \infty$, the mean attrition length scales as $\langle L\rangle \sim N^{1-1 / k_{0}}$, which coincides with the result for networks with short-tailed degree distribution. In the limit of large $k_{0}$, we have $\langle L\rangle \sim N$, i.e., KGWs can continue without being blocked until reaching a length on the order of the system size.
Shown in Fig. 8 is the mean attrition length vs mean connectivity $\langle k\rangle$ for the same networks considered in Fig. 7 , with a size $N=64000$. In this case, differences between results for different kinds of networks are larger than for the self-intersection length (note the logarithmic scale for $\langle L\rangle$ in Fig. 8). In general, for fixed $N$ and $\langle k\rangle$, dispersion in the connectivity distribution (i.e., increase in $\left\langle k^{2}\right\rangle$ ) entails a decrease in $\langle L\rangle$, as observed for the three kinds of networks considered here. If we look at the change of the mean attrition length with $\langle k\rangle$, we find for networks with short-tailed degree distribution (including those with constant degree), that $\langle L\rangle$ increases as $\langle k\rangle$ rises. For these networks, increasing $\langle k\rangle$ makes less probable the appearance of blocking nodes (an escape way is more easily found), and KGWs can continue further.

For scale-free networks, however, $\langle L\rangle$ decreases as $\langle k\rangle$ is raised for constant network size. This is a consequence of the fact that an increase in mean connectivity is associated to a decrease in the exponent $\gamma$ (results shown as triangles in Fig. 8 correspond to scale-free networks with $\gamma$ ranging between 5 and 2 , from left to right). The large degree dispersion in scale-free networks, and the concomitant appearance of nodes with connectivity much larger than the average value $\langle k\rangle$, causes an increase in $\left\langle k^{2}\right\rangle$ and in consequence a decrease in $\langle L\rangle$. This decrease is not observed for the short-tailed degree distributions studied above, since in this case the number $N_{k_{0}}$ of nodes with the lowest degree is reduced very fast for increasing $\langle k\rangle$. In fact, one has $N_{k_{0}} / N=e^{-\lambda}$, versus a much slower reduction of $N_{k_{0}}$ with lowering $\gamma$ in scale-free networks. Since nodes with degree $k_{0}$ are most efficient to block KGWs, we observe a change in the trend of $\langle L\rangle$ shown in Fig. 8, when passing from short- to long-tailed degree distributions. This means that the inhomogeneity of the degree distribution is crucial for determining the maximum length of KGWs in random networks.

\section{DISCUSSION}

Self-intersection and attrition lengths in generalized random networks have been calculated by using an approximate probabilistic method, which gives results in line with those derived from network simulations. Both, the average self-intersection length and attrition length scale as a power of the system size $N$. For the different kinds of networks considered here, the mean selfintersection length of non-reversal walks increases for large system size as $\langle l\rangle \sim N^{\beta}$, with an exponent $\beta$ which depends upon the degree distribution. For short-tailed and scale-free distributions with $\gamma>3$ we find $\beta=0.5$, and this exponent decreases for power-law degree distributions with $\gamma<3$. Note, for comparison, that in regular lattices the mean self-intersection length $\langle l\rangle$ has a finite value, independent of the system size (assumed to be large enough) [29]. This is of course due to the presence of loops. Random networks, however, are locally 
tree-like, and $\langle l\rangle$ is controlled by the system size.

The length of KGWs is limited by attrition of the paths. For uncorrelated networks of large enough size, the mean attrition length $\langle L\rangle$ increases with system size as $\langle L\rangle \sim N^{\alpha}, \alpha$ being an exponent which changes markedly with the minimum degree $k_{0}$. For short-tailed and scale-free distributions with $\gamma>3$, we find $\alpha=$ $1-1 / k_{0}$. For scale-free networks with $\gamma<3$, the exponent $\alpha$ is lower, and the efficiency of KGWs to explore random networks, as measured by the number of visited sites, decreases for decreasing $\gamma$. This is a consequence of the inhomogeneity of the degree distribution present in scale-free networks, which in fact reduces the capability of KGWs to explore them effectively. This low effectivity is expected to be even lower for nonequilibrium scale-free networks, such as those proposed by Barabási and Albert [6]. In these networks, the clustering coefficient is much larger than in uncorrelated networks, and one has many more small-size loops than in the networks studied here. This means that in growing nonequilibrium networks the mean self-intersection and attrition lengths will be lower.

A characteristic of SAWs usually studied in regular lattices is the mean squared end-to-end distance, which scales for large length as $n^{2 \nu}, \nu$ being a dimensiondependent critical exponent [29, 35]. For $D>4$ one has $\nu=\frac{1}{2}$, as for unrestricted random walks 47]. For random networks, a true distance is not defined and we consider an end-to-end separation for KGWs, the separation between two nodes being defined as the number of links along the shortest path connecting them. Then, the mean squared end-to-end separation of KGWs on random networks scales as $n^{2}$ in the thermodynamic limit, i.e., with an exponent $\nu=1$ [30]. This exponent coincides with that corresponding to SAWs for $D=1$, because loops become irrelevant in random networks for $N \rightarrow \infty$ (they become tree-like). However, note that this behaviour is not obtained for KGWs on finite networks, for which the mean end-to-end separation converges for large $n$ to a constant on the order of the mean separation between nodes [30].
Another long-range property of SAWs is the connective constant $\mu$. As mentioned above, the number of SAWs on regular lattices scales for large $n$ as $s_{n} \sim n^{\Gamma-1} \mu^{n}$, where $\Gamma$ depends on the lattice dimension $D$, and $\Gamma=1$ for $D>4$ [29, 35]. For random networks we find $s_{n} \sim \mu_{\infty}^{n}$, indicating that $\Gamma=1$, the same exponent as for regular lattices in many dimensions. This contrasts with the exponent $\nu=1$ discussed above for the mean squared end-to-end separation, which coincides with that for $D=1$, and indicates an important difference between random networks and regular lattices in what refers to KGWs. In fact, the absence of loops in random networks for $N \rightarrow \infty$, makes the end-to-end separation equal to $n$, as for a linear lattice. However, for the number of KGWs (and the connective constant $\mu$ ), random networks behave as regular lattices in the limit $D \rightarrow \infty$, where loops, although present, become irrelevant for many purposes.

In summary, kinetic growth walks are well suited for exploring the long-range topology of networks. In the limit of large random networks, the characteristics of these walks can be related with those known for regular lattices. However, finite-size effects are found to be crucial to understand long-range features of KGWs on finite random networks. Inhomogeneity in the degree distribution reduces appreciably the attrition length of these walks, in particular for scale-free networks with exponent $\gamma \leq 3$. This reduction is expected to be even larger for nonequilibrium scale-free networks, as those of Barabási-Albert type.

\section{Acknowledgments}

Thanks are due to M. Saboyá for critically reading the manuscript. This work was supported by CICYT (Spain) under Contract No. BFM2003-03372-C03-03.
[1] S. H. Strogatz, Nature 410, 268 (2001).

[2] S. N. Dorogovtsev and J. F. F. Mendes, Evolution of Networks: From Biological Nets to the Internet and $W W W$ (Oxford University, Oxford, 2003).

[3] R. Albert and A. L. Barabási, Rev. Mod. Phys. 74, 47 (2002).

[4] M. E. J. Newman, SIAM Rev. 45, 167 (2003).

[5] D. J. Watts and S. H. Strogatz, Nature 393, 440 (1998).

[6] A. L. Barabási and R. Albert, Science 286, 509 (1999).

[7] C. Moore and M. E. J. Newman, Phys. Rev. E 61, 5678 (2000).

[8] M. Kuperman and G. Abramson, Phys. Rev. Lett. 86, 2909 (2001).

[9] C. P. Herrero, Phys. Rev. E 66, 046126 (2002).

[10] Y. Moreno, M. Nekovee, and A. F. Pacheco, Phys. Rev. E 69, 066130 (2004).
[11] S. A. Pandit and R. E. Amritkar, Phys. Rev. E 63, 041104 (2001).

[12] J. Lahtinen, J. Kertész, and K. Kaski, Phys. Rev. E 64, 057105 (2001).

[13] M. E. J. Newman, Proc. Natl. Acad. Sci. USA 98, 404 (2001).

[14] H. Jeong, S. P. Mason, A. L. Barabási, and Z. N. Oltvai, Nature 411, 41 (2001).

[15] G. Siganos, M. Faloutsos, P. Faloutsos, and C. Faloutsos, IEEE ACM Trans. Netw. 11, 514 (2003).

[16] R. Albert, H. Jeong, and A. L. Barabási, Nature 401, 130 (1999).

[17] K. I. Goh, E. S. Oh, H. Jeong, B. Kahng, and D. Kim, Proc. Natl. Acad. Sci. USA 99, 12583 (2002).

[18] D. J. Watts, P. S. Dodds, and M. E. J. Newman, Science 296, 1302 (2002). 
[19] P. S. Dodds, R. Muhamad, and D. J. Watts, Science 301, 827 (2003).

[20] R. Guimerà, A. Díaz-Guilera, F. Vega-Redondo, A. Cabrales, and A. Arenas, Phys. Rev. Lett. 89, 248701 (2002).

[21] S. Jespersen and A. Blumen, Phys. Rev. E 62, 6270 (2000).

[22] F. Jasch and A. Blumen, Phys. Rev. E 64, 066104 (2001).

[23] B. Tadić, Eur. Phys. J. B 23, 221 (2001).

[24] J. D. Noh and H. Rieger, Phys. Rev. Lett. 92, 118701 (2004).

[25] B. J. Kim, C. N. Yoon, S. K. Han, and H. Jeong, Phys. Rev. E 65, 027103 (2002).

[26] E. Almaas, R. V. Kulkarni, and D. Stroud, Phys. Rev. E 68, 056105 (2003).

[27] N. Masuda and N. Konno, Phys. Rev. E 69, 066113 (2004).

[28] L. A. Adamic, R. M. Lukose, A. R. Puniyani, and B. A. Huberman, Phys. Rev. E 64, 046135 (2001).

[29] A. D. Sokal, in Monte Carlo and Molecular Dynamics Simulations in Polymer Science, edited by K. Binder (Oxford University, Oxford, 1995), pp. 47-124.

[30] C. P. Herrero, Phys. Rev. E 71, 016103 (2005).

[31] P. G. de Gennes, Scaling Concepts in Polymer Physics (Cornell University, Ithaca, NY, 1979).

[32] S. B. Lee, H. Nakanishi, and Y. Kim, Phys. Rev. B 39, 9561 (1989).

[33] C. P. Herrero, J. Phys.: Condens. Matter 7, 8897 (1995).
[34] K. Kremer, A. Baumgärtner, and K. Binder, J. Phys. A: Math. Gen. 15, 2879 (1982).

[35] V. Privman, P. C. Hohenberg, and A. Aharony, in Phase Transitions and Critical Phenomena, edited by C. Domb and J. L. Lebowitz (Academic Press, London, 1991), vol. 14 .

[36] C. P. Herrero and M. Saboyá, Phys. Rev. E 68, 026106 (2003).

[37] L. da F. Costa, cond-mat/0312712.

[38] M. E. J. Newman, S. H. Strogatz, and D. J. Watts, Phys. Rev. E 64, 026118 (2001).

[39] I. Majid, N. Jan, A. Coniglio, and H. E. Stanley, Phys. Rev. Lett. 52, 1257 (1984).

[40] J. W. Lyklema and K. Kremer, J. Phys. A: Math. Gen. 17, L691 (1984).

[41] S. S. Manna and A. J. Guttmann, J. Phys. A: Math. Gen. 22, 3113 (1989).

[42] G. Bianconi and A. Capocci, Phys. Rev. Lett. 90, 078701 (2003).

[43] B. Bollobás, Modern Graph Theory (Springer-Verlag, New York, 1998).

[44] D. S. Dean and A. Lefèvre, Phys. Rev. Lett. 86, 5639 (2001).

[45] S. Boettcher, Phys. Rev. B 67, 060403 (2003).

[46] M. Molloy and B. Reed, Random Struct. Algorithms 6, 161 (1995).

[47] G. Slade, J. Phys. A: Math. Gen. 21, L417 (1988). 